\title{
THE GALACTIC CENTER
}

"I have a feeling that one does not understand at all how things happen at the center."

J. H. Oort, in the discussion following his paper 


\title{
INFRARED RADIATION FROM THE GALACTIC CENTER
}

\author{
J. BOR GMAN \\ Kapteyn Observatory, Roden, The Netherlands
}

\begin{abstract}
IR observations of the region within 0.5 of $\mathrm{Sgr} \mathrm{A}$ are discussed. The total IR luminosity is $150 \times 10^{6} L_{\odot}$. Most of it is stellar radiation, absorbed by low temperature dust and reradiated in the IR. There is no unique interpretation for the highly structured infrared core. It may comprise compact infrared sources like those in some Hil regions, or the structure may simply result from the dust distribution. Nonthermal radiation is also feasible, but a tradeoff between source luminosity and extinction is possible and prevents a final decision on this question.
\end{abstract}

\section{Introduction}

Since the first papers on infrared radiation from the galactic center by Becklin and Neugebauer $(1968,1969)$, considerable observational work has been done. Observations are now available on the far infrared surface brightness of a $4^{\circ} \times 2^{\circ}$ region (Hoffmann et al., 1971). Fine structure has been mapped within 20" of the near infrared maximum surface brightness by Rieke and Low (1973). The broadband spectrum of a number of features has been determined (Becklin and Neugebauer, 1969; Rieke and Low, 1973; Harper and Low, 1973), and a broad absorption band has been detected near $9.7 \mu \mathrm{m}$ (Woolf, 1973).

The present paper aims to briefly describe and discuss published as well as some new observations. We will largely restrict ourselves to the region within 0.5 of Sgr A. Following the usual practice the observations will be ascribed to 'sources'. This terminology is used rather loosely and is not much more than a convenient way to characterize the observations in terms of the spectral and spatial distribution of the observed radiation.

\section{Observations}

Table I presents a condensed summary of observations which are more fully discussed below.

\section{(a) THE NEAR INFRARED HALO}

Becklin and Neugebauer (1968) mapped the $2.2 \mu \mathrm{m}$ radiation in a region of $30^{\prime} \times 40^{\prime}$ using an aperture of $1.8^{\prime}$; in addition, they obtained maps at $2.2 \mu \mathrm{m}$ of smaller regions with higher resolution. For the central $110^{\prime \prime}$ a study was made of the relation between the field of view and the measured radiation at 1.65, 2.2, 3.5 and $4.8 \mu \mathrm{m}$.

Figure 1 shows the $2.2 \mu \mathrm{m}$ map of Becklin and Neugebauer, obtained with a $1.8^{\prime}$ aperture. The map is dominated by the near infrared nucleus (see Section IIb), the center of which coincides with the radio position of Sgr A. In addition several secondary sources can be seen; none of these secondary sources has been observed at longer wavelengths. The position of the source at $17^{\mathrm{h}} 42^{\mathrm{m}} 44^{\mathrm{s}},-29^{\circ} 00^{\prime}$, is close to the position 
TABLE I

Condensed summary of typical data

\begin{tabular}{|c|c|c|c|c|c|c|c|}
\hline \multirow[t]{2}{*}{ Name } & \multirow[t]{2}{*}{ Area } & $F_{\lambda} \quad$ at & $\lambda$ & $P_{\lambda \lambda}$ & $L \quad$ at & $\lambda \lambda$ & \multirow[t]{2}{*}{ Ref. $^{a}$} \\
\hline & & Jy & $\mu \mathrm{m}$ & $10^{-10} \mathrm{~W} \mathrm{~m}^{-2}$ & $10^{6} L_{\odot}$ & $\mu \mathrm{m}$ & \\
\hline Near infrared halo & $\sim 40^{\prime} \times 30^{\prime}$ & 2000 & 2.2 & 11 & 3 & $1.5-2.4$ & 1 \\
\hline Near infrared halo & $\sim 1^{\circ} \times 1^{\circ}$ & 1000 & 5.5 & 1 & 0.3 & $5-6$ & 2 \\
\hline Near infrared nucleus & $\begin{array}{l}5^{\prime} \times 3^{\prime} \\
\left(10^{\prime} \times 6^{\prime}\right)\end{array}$ & 80 & 2.2 & 1 & 0.3 & $1.5-4.0$ & 1 \\
\hline Near infrared nucleus & $\begin{array}{l}\text { minus } \\
\left(5^{\prime} \times 3^{\prime}\right)\end{array}$ & 100 & 2.2 & 0.6 & 0.2 & $1.5-2.4$ & 1 \\
\hline Near infrared nucleus & $22^{\prime \prime}$ & 5 & 2.2 & 0.1 & $<0.1$ & $1.06-4.0$ & 1,7 \\
\hline Infrared core & $22^{\prime \prime}$ & 900 & 12.2 & 3 & 1 & $3.1-25$ & $1,3,4,5$ \\
\hline Far infrared halo & $38^{\prime} \times 15^{\prime}$ & $1.5 \times 10^{6}$ & 100 & 240 & 74 & $75-125$ & 6 \\
\hline Far infrared halo & $7^{\prime}$ & - & - & 130 & 40 & $10-300$ & 8 \\
\hline
\end{tabular}

a References quoted:

1. Becklin and Neugebauer $(1968,1969) \quad 5$. Low et al. (1969)

2. Houck et al. (1971) 6. Hoffman et al. (1971)

3. Woolf (1973) 7. Spinrad et al. (1971)

4. Borgman $(1972,1973) \quad$ 8. Low and Aumann (1970)

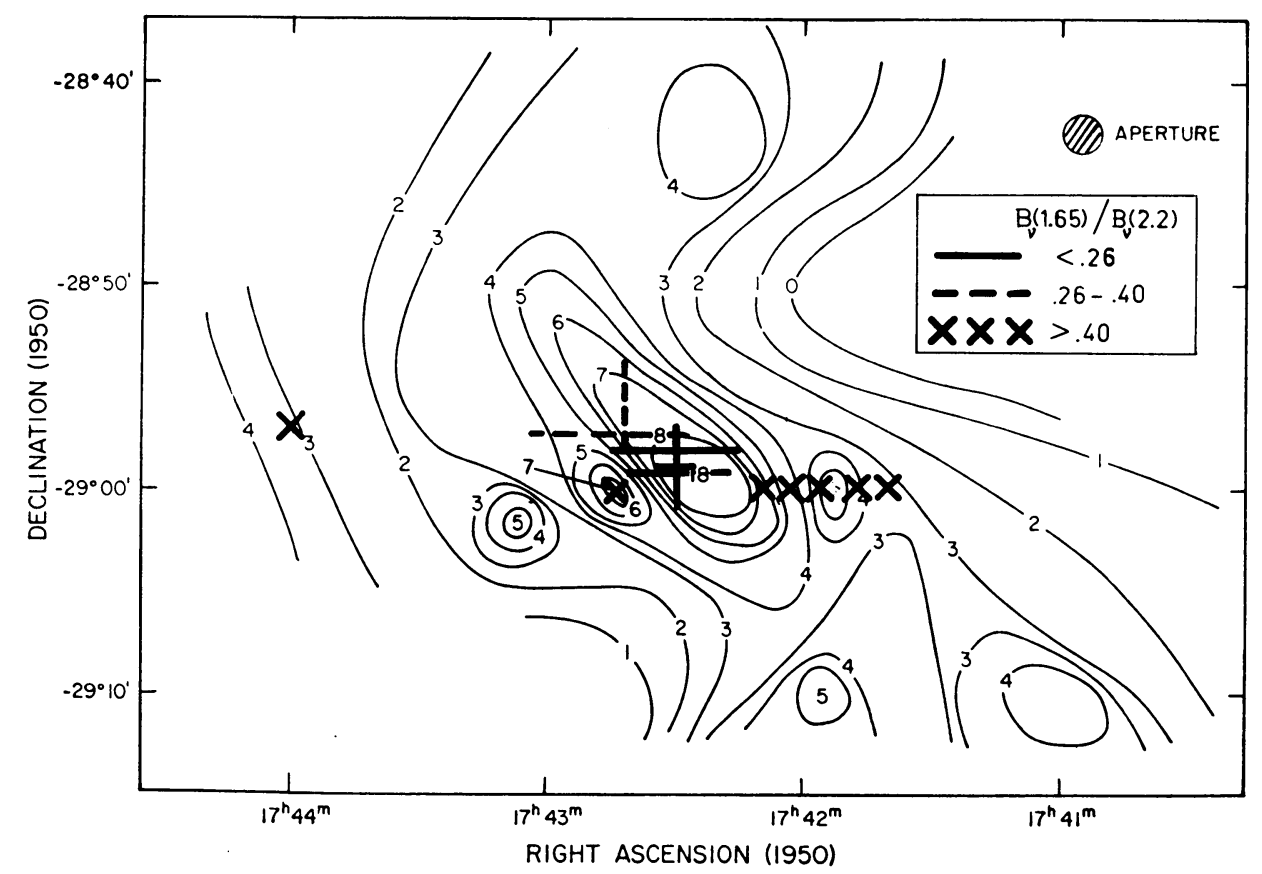

Fig. 1. Becklin and Neugebauer's (1968) contour map at $2.2 \mu \mathrm{m}$ observed with an aperture of $1.8^{\prime}$ diameter. Contour lines are separated by $8.5 \times 10^{-20} \mathrm{~W} \mathrm{~m}^{-2} \mathrm{~Hz}^{-1} \mathrm{~s}^{-1}$ and are uncertain by the same amount. Color information, added to the contour diagram refers to 1.65 and $2.2 \mu \mathrm{m}$ scans (Becklin and Neugebauer, 1968) and is explained in the insert. 
of the $40 \mathrm{~km} \mathrm{~s}^{-1} \mathrm{H}_{2} \mathrm{CO}$ cloud discussed, e.g., by Fomalont and Weliachew (1973). Becklin and Neugebauer made a number of scans and measurements within the area of Figure 1 which indicate that the color gets redder towards the near infrared nucleus. We will adopt $B \lambda(1.65) / B \lambda(2.2)=0.51$ for the near infrared halo, with a flux density of $2000 \mathrm{Jy}$ at $2.2 \mu \mathrm{m}$. The flux density has been derived from a mean brightness of $25 \times 10^{-20} \mathrm{~W} \mathrm{~m}^{-2} \mathrm{~Hz}^{-1} \mathrm{~s}^{-1}$ outside the near infrared nucleus over the 1000 arc$\min ^{2}$ mapped in Figure 1. It is clear that the near infrared halo extends beyond the limits of this map; therefore, the actual $2.2 \mu \mathrm{m}$ flux density will be higher.

\section{(b) THE NEAR INFRARED NUCLEUS}

This is the dominant source of Figure 1, which has been observed at wavelengths $1.65,2.2,3.5,4.8$ and $10.1 \mu \mathrm{m}$. The observations at 4.8 and $10.1 \mu \mathrm{m}$ will be dealt with under the infrared core (see Section IIc), together with the $20 \mu \mathrm{m}$ observations.

The near infrared nucleus has been observed in considerable detail at $2.2 \mu \mathrm{m}$ by Becklin and Neugebauer (1968). They found that the $2.2 \mu \mathrm{m}$ radiation distribution had a dominant source at $17^{\mathrm{h}} 42^{\mathrm{m}} 30^{\mathrm{s}} \pm 1^{\mathrm{s}},-28^{\circ} 59^{\prime} .4 \pm 0.1$ with a full width at half maximum of $3^{\prime} \times 5^{\prime}$ when observed with a $1.8^{\prime}$ aperture. This source has a $2.2 \mu \mathrm{m}$ angular distribution which is characterized by a power law such that the flux density within a circular aperture of diameter $D$ is proportional to $D^{1.2 \pm 0.1}$, corresponding to a mean surface brightness which is proportional to $D^{-0.8 \pm 0.1}$. This power law holds in the aperture range $5^{\prime \prime}-10^{\prime}$. The same dependence on aperture has been observed at $1.65 \mu \mathrm{m}$ over the aperture range $16^{\prime \prime}-110^{\prime \prime}$. According to Becklin and Neugebauer (1968), the near infrared nucleus has a color $B \lambda(1.65) / B \lambda(2.2)=0.20$. In our discussion we will adopt this figure with the restriction that it is valid only for the central $5^{\prime} \times 3^{\prime}$ part. Somewhat arbitrarily we adopt for our discussion $B \lambda(1.65) /$ $B \lambda(2.2)=0.35$ for the annulus $\left(10^{\prime} \times 6^{\prime}\right)-\left(5^{\prime} \times 3^{\prime}\right)$, a value intermediate between the color of the center and the color of the near infrared halo (see Section IIa).

Spinrad et al. (1971) detected the near infrared nucleus at 1.06 and $1.18 \mu \mathrm{m}$, with measurements at the position of the maximum $2.2 \mu \mathrm{m}$ brightness with a $22^{\prime \prime}$ diaphragm.

\section{(c) THE INFRARED CORE}

Becklin and Neugebauer (1969) and Low et al. (1969) made the first measurements of a small source $\left(d \approx 16^{\prime \prime}\right)$ which emits most of the 10 and $20 \mu$ m radiation of the galactic center. Ground-based observing techniques do not exclude the existence of a more extended 10 and $20 \mu \mathrm{m}$ source of low surface brightness; however, rocket observations by Houck et al. (1971) make it unlikely that a 1' aperture would contain less than half of the total radiation at these wavelengths (cf. Rieke and Low's data for a $1^{\prime}$ aperture).

The position of the $16^{\prime \prime}$ source coincides with the center of the near infrared nucleus at $2.2 \mu \mathrm{m}$. In addition, Becklin and Neugebauer (1968) found a point-like source at less than $10^{\prime \prime}$ from the center of the $16^{\prime \prime}$ source.

Recently, Rieke and Low (1973) obtained detailed maps of $\sim 30^{\prime \prime} \times 30^{\prime \prime}$ area at 


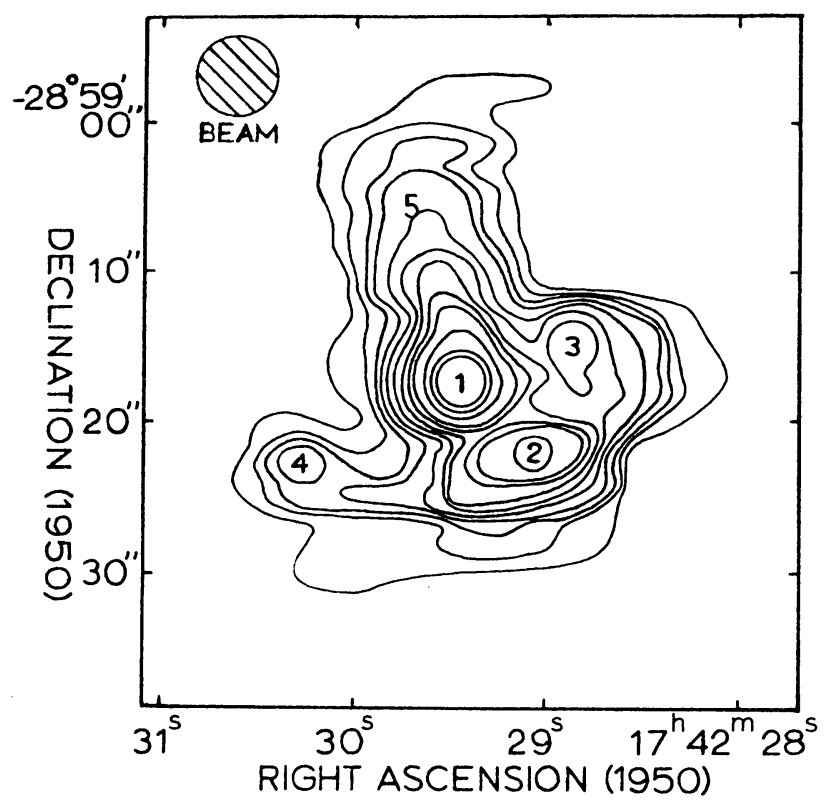

Fig. 2. Rieke and Low's (1973) $10.5 \mu \mathrm{m}$ map of the infrared core. The contours are at $1,2,3,4,6,8,10,12$, $14,16,18,20,22,24$ and 26 times $7.6 \times 10^{-17} \mathrm{~W} \mathrm{~m}^{-2} \mathrm{~Hz}^{-1} \mathrm{~s}^{-1}$. The position of source 5 has been copied from Rieke and Low's (1973) $5 \mu \mathrm{m}$ map.

3.5, 5.0, 10.5 and $21 \mu \mathrm{m}$. Their maps show five sources, four of which can be seen in the $10.5 \mu \mathrm{m}$ map reproduced in Figure 2.

Woolf (1973) published a scan of a strong and broad absorption feature at $9.7 \mu \mathrm{m}$. The profile was measured with a $22^{\prime \prime}$ diaphragm which possibly encircles the sources 1 and 2 of Figure 2; these two sources largely determine the position of the $10 \mu \mathrm{m}$ centroid. Borgman (1973) measured the depth of the $9.7 \mu \mathrm{m}$ absorption feature at the position of the four sources in Figure 2 separately. The following discussion summarizes the results of these studies.

(i) The pointlike source. Following Rieke and Low (1973), we will identify the pointlike source (Becklin and Neugebauer, 1968) with source 3, discussed under Section IIc (iv).

(ii) The $16^{\prime \prime}$ source. We will assume that the $16^{\prime \prime}$ source (5" south and $2^{\prime \prime}$ east of the pointlike source according to Becklin and Neugebauer, 1969) is a blend of sources 1 and 2 in Figure 2. This expectation is supported by a comparison of the observed flux density (Becklin and Neugebauer, 1969) and an integration of the brightness over a circular 16" diaphragm in Figure 2. The positional agreement between Becklin and Neugebauer (1969) and Rieke and Low (1973) is, however, not entirely convincing.

(iii) Sources 1 and 2. These two sources are the major features of the $10.5 \mu \mathrm{m}$ map in Figure 2. At $21 \mu \mathrm{m}$ Rieke and Low (1973) observed a single concentration, coin- 
ciding with the position of source 1 . The contribution of source 2 to the $21 \mu \mathrm{m}$ map is difficult to estimate. Rieke and Low correct their data for 29 mag of visual extinction, resulting in intrinsic color temperatures of approximately $350 \mathrm{~K}$.

Borgman (1973), observing sources 1 and 2 with $0.7 \mu \mathrm{m}$ bandwidth filters and a 7" diaphragm finds [8.1-12.2] color temperatures of $275 \pm 20 \mathrm{~K}$ and $250 \pm 20 \mathrm{~K}$, resp. The depth of the $9.7 \mu \mathrm{m}$ absorption feature, when measured with a $1.0 \mu \mathrm{m}$ bandwidth filter, is 1.7 and $2.0 \mathrm{mag}$, resp. These differences are considered real. The difference with Rieke and Low's color temperatures may be partly due to Rieke and Low's neglecting the $9.7 \mu \mathrm{m}$ depression of approximately 0.4 mag when observing with a wide-band $10 \mu \mathrm{m}$ filter. The influence of a correction of $29 \mathrm{mag}$ of visual extinction on a [8.1-12.2] color temperature of $250 \mathrm{~K}$ is only $10 \mathrm{~K}$.

Sources 1 and 2 are the dominant features of the infrared core at $10 \mu \mathrm{m}$. This suggests that the profile of the $9.7 \mu \mathrm{m}$ depression as published by Woolf (1973) is typical for Sources 1 and 2 .

(iv) Source 3. The broadband spectrum between $1.65 \mu \mathrm{m}$ and $5 \mu \mathrm{m}$, corrected for $29 \mathrm{mag}$ of visual extinction, fits a temperature of $2000 \mathrm{~K}$ (Rieke and Low). The 10 and $20 \mu \mathrm{m}$ observations show a considerable excess over the $2000 \mathrm{~K}$ curve, suggesting that at these wavelengths Source 3 is lost in the background. This suggestion is confirmed by our 7" data, which indicate a [8.1-12.2] color temperature of $275 \pm 30 \mathrm{~K}$. With the same 7" diaphragm we measured the depth of the $9.7 \mu \mathrm{m}$ feature, which turns out to be $2.3 \mathrm{mag}$. For a fuller discussion of the pointlike object we refer to Becklin and Neugebauer (1968).

(v) Source 4. Our 7" aperture data indicate a color temperature of $200 \pm 20 \mathrm{~K}$ at the position of source 4 . The $9.7 \mu \mathrm{m}$ depression is $2.1 \mathrm{mag}$ (mean value of two discordant observations) when measured with a $1.0 \mu \mathrm{m}$ bandwidth filter. Source 4 shows only up on the $10.5 \mu \mathrm{m}$ map of Rieke and Low; at other wavelengths there are no closed contours at the position of this source. We suggest that Source 4 can be regarded as part of the diffuse radiation in the infrared core.

(vi) Source 5. This source shows only up on the $5 \mu \mathrm{m}$ map of Rieke and Low, where it is about as strong as Source 3. At 3.5, 10.5 and $21 \mu \mathrm{m}$ there is no evidence for the existence of Source 5.

(d) THE FAR INFRARED HALO

Hoffman et al. (1971) mapped the galactic center region, using a $12^{\prime}$ aperture and a 75-125 $\mu \mathrm{m}$ bandpass. They detected at least three discrete sources and an extended background of $4^{\circ} \times 2^{\circ}$. The observed luminosity in this area (which includes Sgr A, Sgr B2 and G359.4-0.1) is $3.4 \times 10^{8} L_{\odot}$.

Hoffmann et al. have adjusted the position of their largest and most luminous source to the radio coordinates of Sgr A. The identification is supported by the relative positions of the two other sources, which then coincide with Sgr B2 and G359.4-0.1. 
Our discussion will be limited to the source identified with $\mathrm{Sgr}$ A. This source measures $38^{\prime} \times 15^{\prime}$ full width at half maximum, and its observed $75-125 \mu \mathrm{m}$ luminosity is $7.4 \times 10^{7} L_{\odot}$. Low and Aumann (1970) studied the dependence of the $50-350$ $\mu \mathrm{m}$ flux density on the field of view; they found that the brightness decreased according to a $D^{-1.1 \pm 0.2}$ power law in a 3 to $14^{\prime}$-aperture range. With a $5^{\prime}$ aperture Harper and Low (1973) observed at 56, 68, 91 and $105 \mu \mathrm{m}$; the flux density seems to peak at $\sim 80 \mu \mathrm{m} .^{*}$

\section{Discussion}

The main conclusions of the discussion of the observations are given in Table II and Figure 3.

Following Becklin and Neugebauer (1968) we will interpret the near infrared radiation of the galactic nucleus as stellar, with a population as suggested by the broadband spectrum of the nucleus of M31 (Sandage et al., 1969): blackbody radiation characterized by a temperature of $4000 \mathrm{~K}$. Assuming the interstellar extinction law to be the same as in the solar neighbourhood (Johnson, 1965) they interpreted the [1.65-2.2] color index as being reddened by $1.9 \mathrm{mag}$, leading to a visual extinction of 27 mag. Essentially the same result was found by Spinrad et al. (1971) and Borgman (1972) when observations at 1.06 and $1.18 \mu \mathrm{m}$ were included.

Actually the figure of $A_{V}=27$ mag was derived for the near infrared nucleus. However, for the near infrared halo it has been argued in Section IIa that the [1.65-2.2] color is 1.0 mag bluer than in the near infrared nucleus. This leads to $A_{V}=13$ mag and a luminosity of $120 \times 10^{6} L_{\odot}$. It should be noted that the $5.5 \mu \mathrm{m}$ flux density of Table I is somewhat but not significantly below the expected value. As the $5.5 \mu \mathrm{m}$

\section{TABLE II}

Interpretation

\begin{tabular}{|c|c|c|c|c|c|c|}
\hline Name & Intrinsic source & $\begin{array}{l}A_{V} \\
\operatorname{mag}\end{array}$ & $\begin{array}{l}\lambda \\
\mu \mathrm{m}\end{array}$ & $\begin{array}{l}A_{\lambda} \\
\operatorname{mag}\end{array}$ & $\begin{array}{l}F_{\lambda}^{\circ} \\
\mathrm{Jy}\end{array}$ & $\begin{array}{l}L_{\text {tot }} \\
10^{6} L_{\odot}\end{array}$ \\
\hline Near infrared halo & stellar, $4000 \mathrm{~K} \mathrm{BB}$ & 13 & 2.2 & 1.2 & 6600 & 120 \\
\hline Near infrared nucleus $5^{\prime} \times 3^{\prime}$ & stellar, $4000 \mathrm{~K} \mathrm{BB}$ & 27 & 2.2 & 2.7 & 1000 & 18 \\
\hline $\begin{array}{l}\text { Near infrared nucleus } \\
\left(10^{\prime} \times 6^{\prime}\right)-\left(5^{\prime} \times 3^{\prime}\right)\end{array}$ & stellar, $4000 \mathrm{~K}$ BB & 20 & 2.2 & 2.0 & 600 & 12 \\
\hline " Near infrared nucleus, $22^{\prime \prime}$ & stellar, $4000 \mathrm{~K} \mathrm{BB}$ & 27 & 2.2 & 2.7 & 60 & 1 \\
\hline a Near infrared nucleus $22^{\prime \prime}$ & stellar, $4000 \mathrm{~K}$ BB & 75 & 4.8 & 1.9 & 200 & 10 \\
\hline a Infrared core, $22^{\prime \prime}$ & diluted $270 \mathrm{~K} \mathrm{BB}$ & 27 & 12.2 & 0.2 & 1100 & 1 \\
\hline Far infrared halo $38^{\prime} \times 15^{\prime}$ & dust & 13 & 100 & 0 & $1.5 \times 10^{6}$ & 150 \\
\hline
\end{tabular}

a These are three alternatives to explain the radiation from the central $22^{\prime \prime}$ central region.

* The agreement between the published photometric quantities of Hoffman et al. (1971), Harper and Low (1973), Aumann and Low (1970) and Low and Aumann (1970) is not very satisfactory; without full information on actually observed data and reduction procedures it is difficult to make a judicious assessment. A preliminary comparison indicates that the flux data of Hoffmann et al. (1971) may be too high, a conclusion which is supported by some recent results of the group at the University of Groningen (Olthof, private communication). 


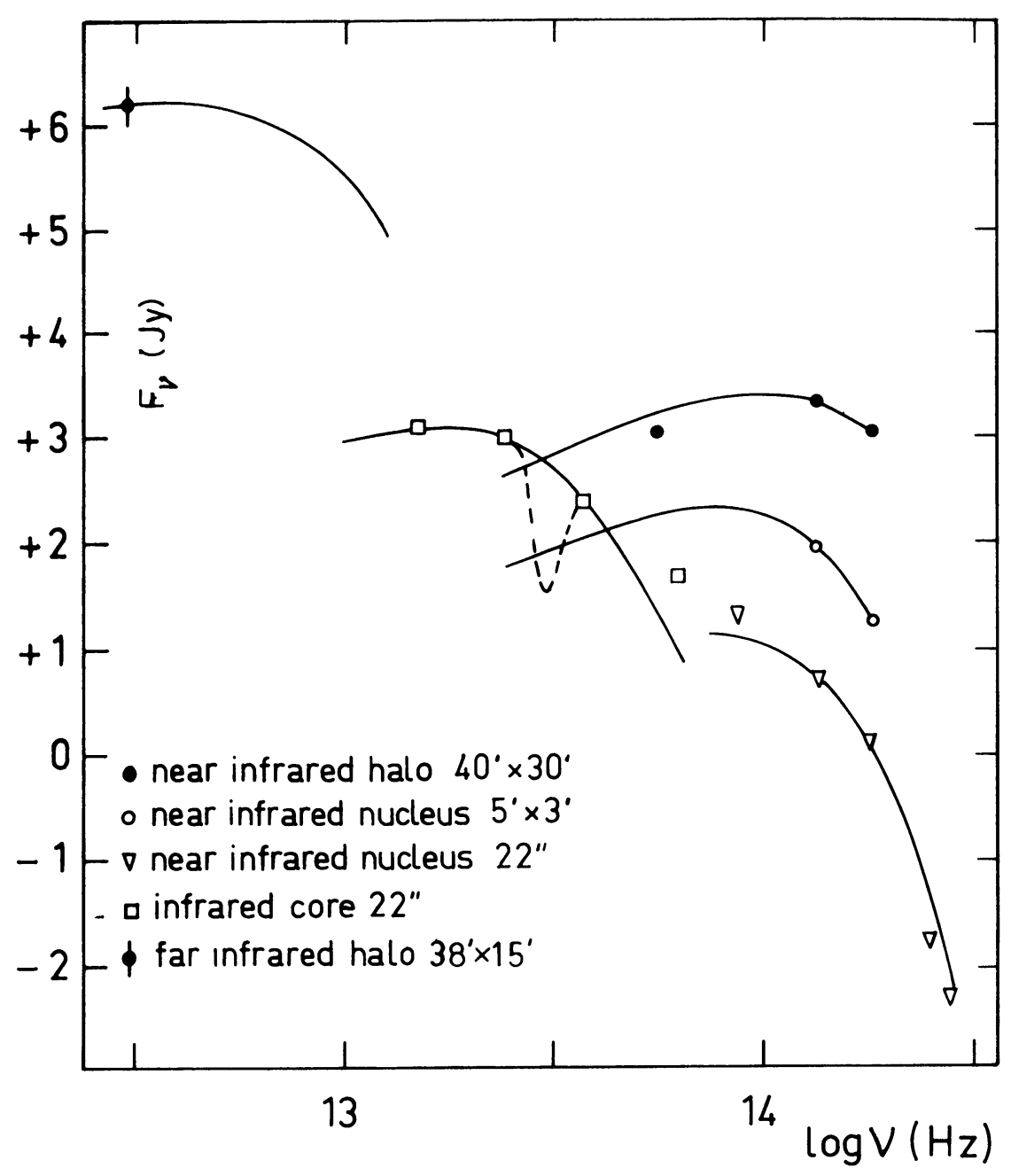

Fig. 3. Spectral energy distribution of various regions in the galactic center. Plotted points are observations taken from the literature quoted in Table $\mathrm{I}$. The dashed curve is the $9.7 \mu \mathrm{m}$ absorption feature, adapted from Woolf (1973). Full drawn curves refer to the interpretation in Table II. The curve fitted to the infrared core data is for a $270 \mathrm{~K}$ black-body and $A_{V}=27 \mathrm{mag}$. For reference, a $65 \mathrm{~K}$ black-body curve has been drawn through the $100 \mu \mathrm{m}$ point of the far infrared halo, but this temperature is certainly too high for the outlying portions of the halo (see text).

flux has been measured for a larger area than is interpreted here we tentatively conclude that the near infrared halo does not extend much beyond the $1000 \mathrm{arcmin}^{2}$ mapped by Becklin and Neugebauer (1968); this conclusion should not be given too much weight as some unpublished uncertainties and observing constraints could not be taken into account.

The adopted $A_{V}=27 \mathrm{mag}$ for the $5^{\prime} \times 3^{\prime}$ near infrared nucleus leads to a luminosity of $18 \times 10^{6} L_{\odot}$. Adopting an intermediate extinction $A_{V}=20$ mag for the annulus 
$(10 \times 6) \operatorname{arcmin}^{2}-(5 \times 3) \operatorname{arcmin}^{2}$ we find a projected luminosity of $12 \times 10^{6} L_{\odot}$. With a simple spherical mass distribution model and adopting $M / L=3$ it can be shown that the total stellar luminosity within the $1000 \mathrm{arcmin}^{2}$ area is approximately $140 \times 10^{6} L_{\odot}$ at a total mass of $4 \times 10^{8} M_{\odot}{ }^{*}$ An inspection of Table I shows that only $5 \%$ of this radiation is observed as direct starlight in the near infrared; $130 \times 10^{6}$ $L_{\odot}$ are available for redistribution over other parts of the spectrum.

The far infrared halo and the near infrared halo have approximately the same brightness distribution, suggesting that they occupy the same volume in space. The total infrared luminosity of the far infrared halo may be a factor 2 larger than the 75-125 $\mu \mathrm{m}$ luminosity as follows from the data in Table I and the flux-aperture relation determined by Low and Aumann (1970). The resulting luminosity of $150 \times 10^{6}$ $L_{\odot}$ is in good agreement with the assumption that $130 \times 10^{6} L_{\odot}$ of stellar energy from the near infrared halo are reradiated by dust in the far infrared. Also the agreement between the data in the smaller fields of $10^{\prime} \times 6^{\prime}\left(30 \times 10^{6} L_{\odot}\right.$ stellar luminosity) and $7^{\prime}$ diameter $\left(40 \times 10^{6} L_{\odot}\right.$ total infrared radiation ascribed to dust $)$ is quite satisfactory and supports the local energy conversion hypothesis. The agreements are somewhat fortuitous (but how much so is difficult to estimate) in view of considerable uncertainties in relation to the data and the somewhat different areas which were compared.

The flux density peak at $80 \mu \mathrm{m}$ (Harper and Low, 1973) suggests a color temperature of $65 \mathrm{~K}$ for the central $5^{\prime}$ of the far infrared halo, but taking into account a diluted spectrum at low frequencies this value may be closer to $45 \mathrm{~K}$ (Andriesse and Olthof, 1973).

The infrared core, radiating predominantly at 10 and $20 \mu \mathrm{m}$, is not well understood and as a consequence has aroused much speculation. Okuda and Wickramasinghe (1970) have proposed a strong concentration of the dust towards the center where they situated a powerful $5 \times 10^{4} \mathrm{~K}$ blackbody. The origin of this source remains unspecified. A variety of nonthermal mechanisms has been discussed by Burbidge and Stein (1970). Lynden-Bell and Rees (1971) suggest accretion of gas by a black hole, resulting in an efficient conversion of the rest-mass energy into UV and optical radiation which is then available for the ionization of the thermal radio source and heating of the dust in the extended far infrared halo.

The $9.7 \mu \mathrm{m}$ absorption feature of the infrared core has been studied in detail by Aitken and Jones (1973), who predict 75 mag of visual extinction. The extinction value depends fully on the adopted extinction in front of the Becklin/Neugebauer object in the Orion nebula and has to be revised if the criticism of Becklin et al. (1973) proves to be right.

Borgman (1972) proposed a dense $1 \mathrm{pc}$ diameter star cluster with a luminosity of $5 \times 10^{8} L_{\odot}$, sufficient to excite all of the observed far infrared emission in an area of $4^{\circ} \times 2^{\circ} ; 200$ mag of visual extinction between the source and the Sun are needed to

\footnotetext{
* As a consequence of adopting a bluer color for the near infrared halo this mass is lower than the values discussed by Sanders and Lowinger (1972), which include those derived earlier by Oort (1971) and Becklin and Neugebauer (1968).
} 
explain the $5-20 \mu \mathrm{m}$ spectrum as an absorption spectrum, but this requires the intervening dust cloud to be at a distance of at least $5 \mathrm{pc}$ in order to keep the dust radiation at $20 \mu \mathrm{m}$ at a tolerable level (Visser, private communication). However, the $2.2 \mu \mathrm{m}$ radiation shows a $1 \mathrm{pc}$ wide peak at the position of the infrared core (Becklin and Neugebauer (1968)); if this is interpreted as radiation from the near infrared nucleus stellar population, which can be seen within $0.5 \mathrm{pc}$ from the center, it is suggested that the postulated dense dust cloud is not larger than $1 \mathrm{pc}$ and coincides with the infrared core. Taking these considerations into account we find that a reduced cluster luminosity of $10^{7} L_{\odot}$ (rather, the central peak in the continuous distribution of stars in the near infrared halo and nucleus) embedded in 75 mag of visual extinction reproduces the 3.5 and $4.8 \mu \mathrm{m}$ data; the excess radiation at $8-20 \mu \mathrm{m}$ in the central 1 pc region $\left(21^{\prime \prime}\right)$ can be ascribed to the high temperature dust in that region, adopting reasonable absorption efficiencies. It should be stressed that the choice of source luminosity and extinction is rather arbitrary and that trade-offs between these two parameters are quite feasible if dust radiation is allowed for. It is not possible, on the basis of now available observations, to decide convincingly between radiation contributions of hot dust and partially obscured sources. The only hard fact is the observed luminosity of $10^{6} \mathrm{~L}_{\odot}$, which is a lower limit as it does not include any far infrared contribution where there are no high angular resolution data.

There is some evidence that the infrared core sources 1 and 2 (and possibly 5) as discussed in Section IIc can be identified in the $8085 \mathrm{MHz}$ map of Balick and Sanders (Gordon, 1974), which would mean that these sources are real and are not the result of extinction windows. Both sources show the $9.7 \mu \mathrm{m}$ feature in absorption, which invites a comparison with the Becklin and Neugebauer source in the Orion nebula (Gillett and Forrest, 1973) and IRS5 in W3 (Wynn-Williams et al., 1973; Aitken and Jones, 1973). However, there is at least one important difference: the luminosity of the galactic core is two orders of magnitude greater. Some 40 sources like W3-IRS5 would be required to explain the $10-20 \mu \mathrm{m}$ data. It should be noted that the near infrared data cannot supply us with a lower estimate of extinction on the basis of an extrapolation of a flat radiospectrum, as the near infrared halo stars (and perhaps hot dust in the dense center) form a comparatively bright foreground.

It is of some interest to consider the contribution to the radiation field by planetary nebulae, which are known to be good infrared emitters. The spatial distribution of these objects has been discussed by Minkowski (1965), who finds a strong concentration towards the galactic center. Addressing ourselves to the volume of space occupied by the near infrared halo we will make an estimate of the numbers of planetaries required to explain the $10-20 \mu \mathrm{m}$ data. Adopting $10^{4} L_{\odot}$ and $350 \mathrm{~K}$ as a typical luminosity and color temperature for a planetary and assuming that the radiation is evenly divided between the UV and the infrared we would need 1000 planetaries to produce the observed 10-20 $\mu \mathrm{m}$ flux density (5000 Jy according to Houck et al. [1971]; notice that the 10-20 $\mu$ m fluxes of the cold dust and the halo stars have been considered negligible). The UV luminosity would be $5 \times 10^{6} L_{\odot}$, which is compatible with the $10^{7} L_{\odot}$ required to keep the extended thermal region ionized (Lynden- 
Bell and Rees, 1971). The numbers given here suggest a ratio of giant stars to planetaries of $1000: 1$, which is not unreasonable considering the possible genetic relationship between these objects and their relative life span expectations. Again, these numbers are not too meaningful, as a hot dust component and a number of $O$ stars will explain the data just as well. It should be noted that the total luminosity of the region occupied by the dust in the far infrared halo of the galactic center can be understood quite well with the luminosity of the late type stellar population; there is no reason to believe that a large fraction of the dust radiation is caused by heating through direct absorption of UV photons, though this is probably the dominant mechanism in the nearby $\mathrm{H}$ II regions.

\section{Conclusion}

Infrared observations of the galactic center region indicate a total luminosity of $150 \times 10^{6} L_{\odot}$ within $50 \mathrm{pc}$ from the center. Nearly all of this luminosity is of stellar origin, but most of it is absorbed and reradiated by low temperature dust in the far infrared. There is no unique interpretation for the highly structured infrared core. Though the deep $9.7 \mu \mathrm{m}$ absorption feature hints at high local extinction (as seems to occur also in some nearby dense molecular clouds) it is probably premature to accept this parameter as a calibrated measure of extinction. The sources in the infrared core may well be giant examples or clusters of compact infrared sources as found in some $\mathrm{H}$ II regions. The observations do not rule out that the infrared core is simply the structured central peak of the stellar and dust distribution.

Assuming that the mass contribution of the $1 \mathrm{pc}$ infrared core is small and adopting $M / L=3$ we find that the mass within $50 \mathrm{pc}$ from the center is $5 \times 10^{8} M_{\odot}$.

\section{Acknowledgements}

It is a pleasure to thank Dr C. D. Andriesse for a critical reading of an early version of the manuscript. The final paper has benefitted from discussions with Drs Gordon, Greenberg, Mezger, Oort and Wynn-Williams.

\section{References}

Aitken, D. K. and Jones, B.: 1973, Astrophys. J. 184, 127.

Andriesse, C. D. and Olthof, H.: 1973, Astron. Astrophys. 27, 319.

Aumann, H. H. and Low, F. J.: 1970, Astrophys. J. 159, L159.

Becklin, E. E. and Neugebauer, G.: 1968, Astrophys. J. 151, 145.

Becklin, E. E. and Neugebauer, G.: 1969, Astrophys. J. 157, L31.

Becklin, E. E., Neugebauer, G., and Wynn-Williams, C. G.: 1973, Astrophys. J. Letters 182, L7.

Borgman, J.: 1973, in L. Mavridis (ed.), Stars and the Milky Way System, p. 188.

Borgman, J.: 1974, in preparation.

Burbidge, G. R. and Stein, W. A.: 1970, Astrophys. J. 160, 573.

Fomalont, E. B. and Weliachew, L.: 1973, Astrophys. J. 181, 781.

Gillett, F. C. and Forrest, W. J.: 1973, Astrophys. J. 179, 483.

Gordon, M. A.: 1974, this volume, p. 477. 
Harper, D. A. and Low, F. J.: 1973, Astrophys. J. 181, 781.

Hoffman, W. F., Frederick, C. L., and Emery, R. J.: 1971, Astrophys. J. Letters 164, L23.

Houck, J. R., Soifer, B. T., Pipher, J. L., and Harwit, M.: 1971, Astrophys. J. Letters 169, L31.

Johnson, H. L.: 1965, Astrophys. J. 141, 923.

Low, F. J. and Aumann, H. H.: 1970, Astrophys. J. Letters 162, L79.

Low, F. J., Kleinmann, D. E., Forbes, F. F., and Aumann, H. H.: 1969, Astrophys. J. Letters 157 , L97.

Lynden-Bell, D. and Rees, M. J.: 1971, Monthly Notices Roy. Astron. Soc. 152, 461.

Minkowski, R.: 1965, Stars and Stellar Systems 5, 321.

Okuda, H. and Wickramasinghe, N. C. : 1970, Nature 226, 134. ,

Oort, J. H.: 1971, in D. J. K. O'Connell (ed.), Nuclei of Galaxies, Pontificiae Academiae Scientiarum Scripta Varia 35, North-Holland Publishing Co., Amsterdam, p. 321.

Rieke, G. H. and Low, F. J.: 1973, Astrophys. J. 184, 415.

Sandage, A. R., Becklin, E. E., and Neugebauer, G.: 1969, Astrophys. J. 157, 55.'

Sanders, R. H. and Lowinger, T.: 1972, Astron. J. 77, 292.

Spinrad, H., Liebert, J., Smith, H. E., Schweizer, F., and Kuhi, L. V.: 1971, Astrophys. J. $165,17$.

Woolf, N. J. : 1973, in J. M. Greenberg and H. C. van de Hulst (eds.), 'Interstellar Dust and Related Topics', IAU Symp. 52, 485.

Wynn-Williams, C. G., Becklin, E. E., and Neugebauer, G.: 1972, Monthly Notices Roy. Astron. Soc. $160,1$.

\section{J. Borgman}

Kapteyn Observatory,

Mensingheweg 20,

Roden, The Netherlands

\section{DISCUSSION}

Mezger: The far IR halo has an apparent size of $38^{\prime} \times 18^{\prime}$. It thus comprises at least the Hin region G0.2- 0.0 , one of the most powerful $\mathrm{HI}$ regions in the galactic center. You attribute all of the dust heating to radiation of Population II stars. I feel that at least part of the heating must be due to heating by $\mathrm{O}$ stars which ionize the $\mathrm{H}$ II region.

Borgman: This is only a small part, probably; it is, moreover, uncertain whether the two regions coincide spatially.

Radhakrishnan: What happens to the energy absorbed by the dust at the frequency of the absorption dip? For example, is it reradiated at longer wavelengths because the temperature of the dust is low?

Borgman: It is reradiated by the dust, both at longer and shorter wavelengths as the color temperature requires a dust temperature of the order of $200-300 \mathrm{~K}$.

Maxwell: What extinction is now seen in the galactic center, say at $2.2 \mu \mathrm{m}$ ?

Borgman: The answer is whatever you want, almost. In my tables I used the 'official' 27 mag extinction, which means that the $2.2 \mu \mathrm{m}$ extinction using the normalized van de Hulst curve is about one-tenth of that : 2.7 mag. However, you can make almost any tradeoff between source luminosity and extinction in front of it by varying the source temperature. 